\title{
Adenomatoid Odontogenic Tumor: Case Report and Literature Review
}

\author{
Vanessa Espinoza, DDS ${ }^{1}$ and Roberto Ortiz, DDS ${ }^{*}$ \\ ${ }^{1}$ Oral and Maxillofacial Surgeon, Private Practice, Guayaquil, Ecuador \\ ${ }^{2}$ General Dentist, Guayaquil, Ecuador
}

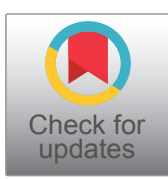

*Corresponding author: Ortiz Roberto, DDS, Los Ceibos, Guayaquil, Ecuador, Tel: +593-99-749-0300

\begin{abstract}
The Adenomatoid Odontogenic Tumor presents in low frequency and is originated from epithelial rests, specifically from the dental lamina, and shows similar characteristics to a wide variety of pathologies. It is a benign non-invasive lesion that can be manage by conservative treatment. Thus, is important to recognize and differentiate it from other pathologies that required more aggressive surgical treatment. The objective of the present article is to report a case of an Adenomatoid Odontogenic Tumor along with its common features.
\end{abstract}

\section{Keywords}

Adenomatoid odontogenic tumor, Odontogenic tumors

\section{Abbreviation}

AOT: Adenomatoid Odontogenic tumor; OT: Odontogenic Tumors

\section{Introduction}

Odontogenic Tumors are a group of heterogenous uncommon lesions that affect maxillary and mandibular osseous tissue, due they originate from mesenchymal and/or epithelial components from the odontogenesis. OT are lesions that present a wide variety of clinical and histopathological features, developing from hamartomas to malignant lesions $[1,2]$.

The AOT was first described more than 100 years ago by Harbitz as "cystic adamantoma". Since then, it has received multiples denominations until 1969 when the World Health Organization accepted the term "Adenomatoid Odontogenic Tumor" proposed by Philipsen and Birn [3]. The WHO defines it as a tumor composed of odontogenic epithelium in a variety of histoarchitec- tural patterns, embedded in a mature connective tissue stroma and characterized by slow but progressive growth [4].

The AOT is the fourth more common odontogenic tumor, appearing in $2-7 \%$ of cases $[5,6]$. It arises from odontogenic epithelium, which indicates it could originate from the enamel organ, reduced enamel epithelium, Mallasez rests or dental lamina rests, being this the most accepted theory [7]. Establishing this pathology as a hamartoma or a neoplasm is reason of great controversy.

Some authors name the AOT as the "two thirds tumor" because two thirds of the cases present in the anterior maxilla, in women, associated with an included tooth of which two thirds is the canine and two thirds of the intraosseous type present radiopacities within the lesion. Tooth resorption is rare; although, cortical expansion and adjacent tooth displacement are regularly seen [8-10].

Three types differentiate according to the location: Follicular and extrafollicular which are differentiated radiographically and peripheral which is observed only clinically [3]. The differential diagnosis with other pathologies such as ameloblastoma and keratocyst is important due to the aggressive treatment they required. Conservative treatment is indicated for AOT because of its low recurrence [6].

The aim of the present article is to report an Adenomatoid Odontogenic Tumor case, as well as describe its more common features allowing correct diagnosis and treatment.

\footnotetext{
Citation: Espinoza V, Ortiz R (2021) Adenomatoid Odontogenic Tumor: Case Report and Literature Review. Res Rep Oral Maxillofac Surg 5:050. doi.org/10.23937/2643-3907/1710050

Accepted: June 21, 2021, 2020; Published: June 23, 2021

Copyright: (C) 2021 Espinoza V, et al. This is an open-access article distributed under the terms of the Creative Commons Attribution License, which permits unrestricted use, distribution, and reproduction in any medium, provided the original author and source are credited.
} 


\section{Case Report}

An asymptomatic 17-year-old female presented to the consult with facial asymmetry on the left side of the maxilla. On the extraoral exploration, an increase of volume of the left maxilla was observed, as well as no change of the skin coloration. Intraorally, it was crackling due to vestibular cortical expansion on left maxilla, extending from the left lateral incisor to the left molar region. Displacement of the left lateral incisor, absence of the left upper canine and persistence of the deciduous canine was also seen.

In the computed tomography was observed a $4 \mathrm{~cm}$ hypodense, unilocular, well-defined and non-invasive image located on the left maxilla. A hyperdense image, compatible with unerupted left canine was noted within the lesion (Figure 1), and displacement of the maxillary sinus.

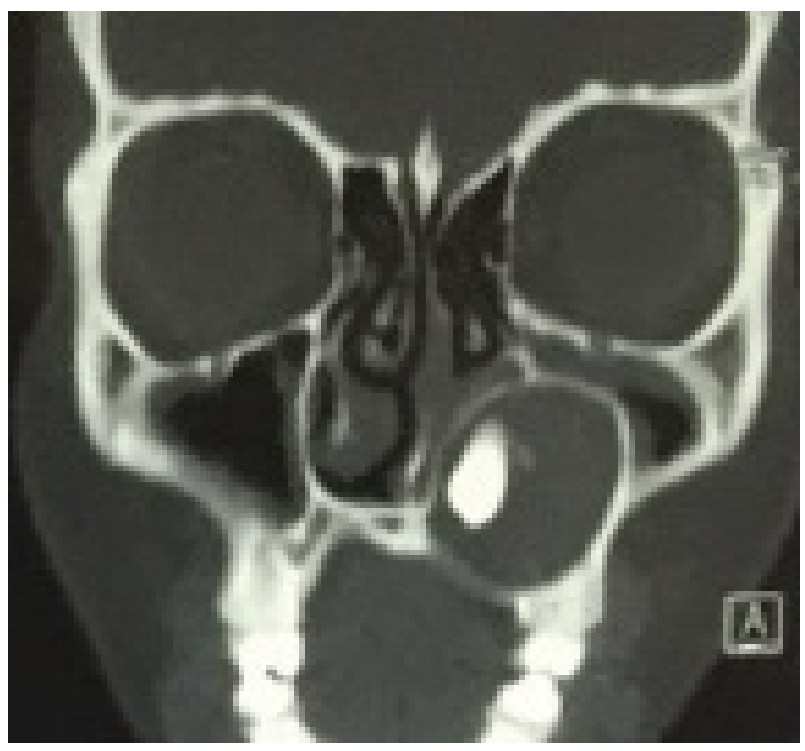

Figure 1: Hyperdensity within unilocular well-defined hypodense image on the left maxilla. View in a coronal section.

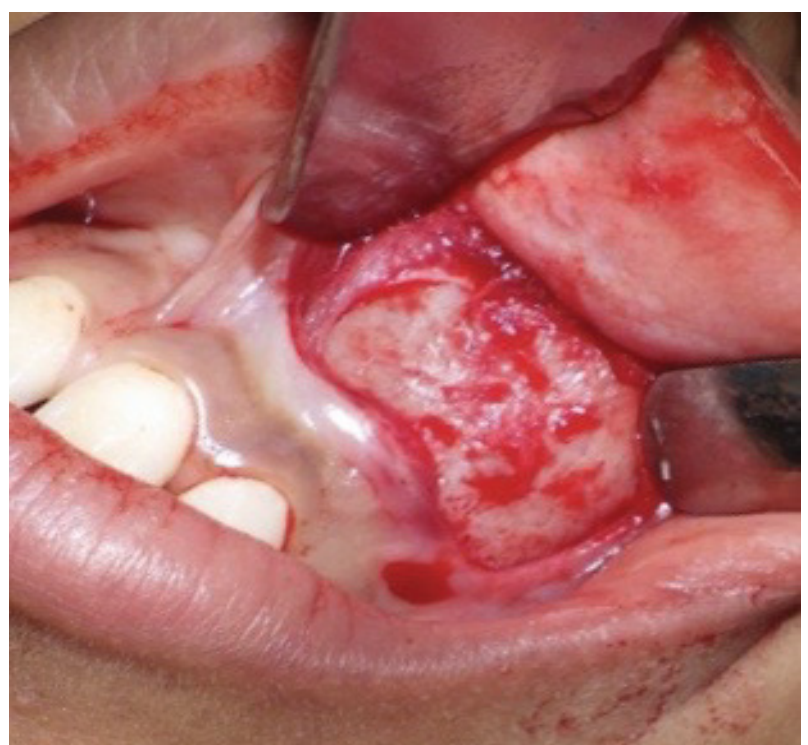

Figure 2: Tumor exposure through vestibular incision.
The treatment, initially, consisted of an incisional biopsy which histopathologic study reported the definitive diagnosis of Adenomatoid Odontogenic Tumor. Posteriorly, under general anesthesia, enucleation and curettage was performed as definitive treatment (Figure 2 and Figure 3).

At 4-year follow-up, the patient showed no signs of clinical or imaging recurrence (Figure 4).

\section{Discussion}

The AOT is an uncommon tumor that originates from the odontogenic epithelium; specifically, from the rests of the dental lamina, which are found in bone, mucosa and the gubernaculum canal. There are three variants depending on the location: follicular, extrafollicular and peripheral $[7,11]$.

The intraosseous variants (follicular and extrafollicular) correspond to $96 \%$ of the cases [12]. The follicular variant is associated to the gubernaculum canal, which

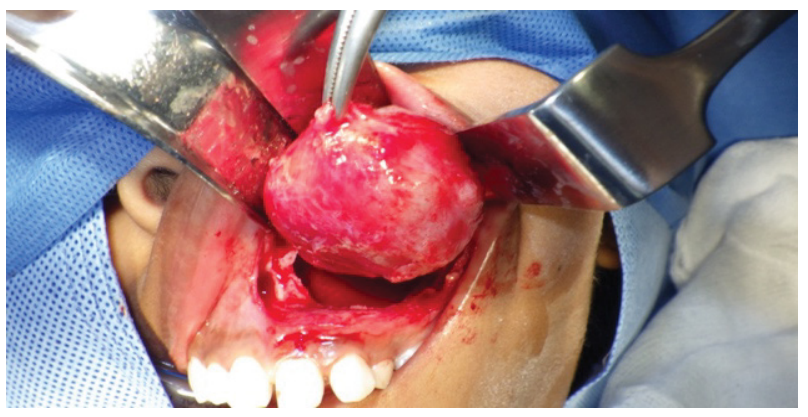

Figure 3: Enucleationy curettage.

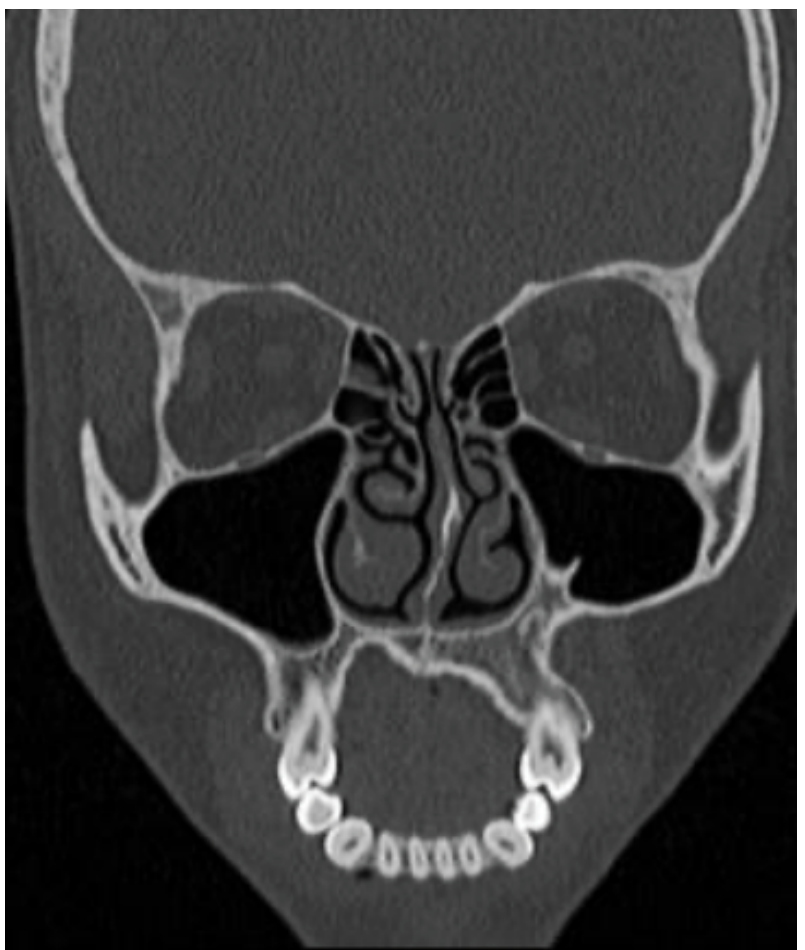

Figure 4: Image in a coronal section shows no sign of recurrence. 
contains a fibrous band with rests of the dental lamina, connecting the peri coronal follicle of a permanent tooth with the alveolar mucosa. In occasions, the rests of the dental lamina from the deciduous teeth can migrate within de gubernaculum canal $[3,7]$. The extrafollicular variant is associated to rests of the dental lamina that remained in the bone after the development of the deciduous germ [7]. The peripheral variant is associated to rests of the dental lamina in the alveolar mucosa and is seeing clinically as a gingival overgrowth $[11,13]$.

Radiographically, the intraosseous variants share some features as well-defined radiolucency with small radiopacities, adjacent tooth displacement, size usually no more than $3 \mathrm{~cm}$; root resorption and cortical perforation are rare [12]. Despite their similarities, the follicular type is associated to an included tooth, being the canine the more frequent; while the extrafollicular type is not associated to an included tooth and can appear between, above, below or superimposed to the roots of permanent teeth [10]. In suspicion of presence of AOT, periapical radiography shows better the calcified deposits within the lesion $[8,13]$.

Clinically, is an expansive and painless lesion that usually displace adjacent teeth. It is a slow growing lesion that shows facial asymmetry when growth is considerable [14]. In some case reports, facial asymmetry caused by growth of the tumor has been compared to an ameloblastoma [15-17].

Classify the AOT as a hamartoma $[8,11,18]$ or as a non-aggressive, non-invasive neoplasm $[5,13,19]$ has generated great controversy. By definition, a neoplasm has unlimited potential for growth; while, the hamartoma has limited potential for growth and is a developmental anomaly with tissue differentiation [13].

The differential diagnosis of the AOT depends on the variant found. The peripheral variant is differentiated only with gingival pathologies such as epulis and gingival fibroma. The diagnosis is purely histopathological $[8,13]$. As most of the cases of the AOT are associated to an unerupted tooth, the differential diagnosis most include the dentigerous cyst; nonetheless, when the radiolucency exceeds the cementum-enamel junction it has to be ruled out $[10,19]$. Because of the small radiopacities within the lesion, the epithelial odontogenic tumor and the epithelial odontogenic cyst should be considered [14]; as well as the ossifying fibroma and cemento-ossifying fibroma. Presenting as a unilocular radiolucency it could appear as an unicystic ameloblastoma. On the other hand, though rare, the AOT can present as a multilocular radiolucency, hence the keratocyst and ameloblastoma most be contemplated [10].

Conservative surgical management consisting of enucleation and curettage is the treatment indicated because of its low recurrence and favorable prognosis $[9,11,14]$.

\section{Conclusion}

The reported case presented the usual features describe in the literature such as age (seventeen), gender (feminine), anatomic region (anterior maxilla), unerupted teeth (canine), slow growing, tooth displacement and no root resorption. Nevertheless, the size was bigger $(4 \mathrm{~cm})$. There was no recurrence after conservative treatment. Identifying the features of this tumor allows differentiating it from other entities that required a different surgical management.

\section{Declarations}

\section{Consent for publication}

Patient's consent for publication was acquired.

\section{Acknowledgements}

Not applicable.

\section{Fundings}

No funding was received.

\section{Competing interests}

The authors declare they have no competing interests.

\section{References}

1. Siriwardena BSMS, Udagama MNGP, Tennakoon TMPB, Athukorala DAADW, Jayasooriya PR, et al. (2020) Clinical and demographic characteristics of adenomatoid odontogenic tumors: analysis of 116 new cases from a single center. Braz J Otorhinolaryngol.

2. Soluk-Tekkesin M, Cakarer S, Aksakalli N, Alatli C, Olgac V (2020) New World Health Organization classification of odontogenic tumours: Impact on the prevalence of odontogenic tumours and analysis of 1231 cases from Turkey. Br J Oral Maxillofac Surg 58: 1017-1022.

3. Philipsen HP, Khongkhunthiang P, Reichart PA (2016) The adenomatoid odontogenic tumour: An update of selected issues. J Oral Pathol Med 45: 394-398.

4. World Health Organization Classification of Tumours (2005) Pathology and genetics of head and neck tumours. Barnes L, Eveson J, Reichart P, Sidransky D, Lyon: IARC Press, 304-305.

5. Suzuki H, Hashimoto K (2005) Adenomatoid Odontogenic Tumour of the Maxilla: Immunohistochemical Study. Asian J Oral Maxillofac Surg 17: 267-271.

6. Sangalette B, Emídio T, Capelari M, Pastori C, Toledo G (2020) Surgical therapy for removal of adenomatoid odontogenic tumor. Hum Pathol Case Rep 20: 200366.

7. Ide F, Mishima K, Kikuchi K, Horie N, Yamachika S, et al. (2011) Development and Growth of Adenomatoid Odontogenic Tumor Related to Formation and Eruption of Teeth. Head Neck Path 5: 123-132.

8. Philipsen HP, Reichart PA (1999) Adenomatoid odontogenic tumour: Facts and figures. Oral Oncol 35: 125-131.

9. Woo VI, Chi AC, Neville BW (2021) Odontogenic Cysts and Tumors. In: Gnepp's Diagnostic Surgical Pathology of the Head and Neck. Elsevier 827-880. 
10. Rajendra Santosh AB, Coard KCM, Williams EB, Jones T (2017) Adenomatoid odontogenic tumor: Clinical and radiological diagnostic challenges. J Pierre Fauchard Acad India Sect 31: 115-120.

11. Díaz Castillejos R, María Nieto Munguía A, Castillo Ham G (2015) Adenomatoid odontogenic tumor. Case report and literature review. Rev Odontológica Mex 19: 183-187.

12. Bartake AR, Punnya VA, Sudeendra P, Rekha K (2009) Two adenomatoid odontogenic tumours of the maxilla: $A$ case report. Br J Oral Maxillofac Surg 47: 638-640.

13. Mohamed A, Singh AS, Raubenheimer EJ, Bouckaert MMR (2010) Adenomatoid odontogenic tumour: review of the literature and an analysis of 33 cases from South Africa. Int J Oral Maxillofac Surg 39: 843-846.

14. Prakasam M, Tiwari S, Satpathy M, Banda VR (2013) Adenomatoid odontogenic tumour. Case Rep.
15. Raubenheimer EJ, Seeliger J, van Heerden W, Dreyer A (1991) Adenomatoid odontogenic tumor: Report of two large lesions. Dentomaxillofac Radiol 20: 43-45.

16. Geist S, Mallon H (1995) Adenomatoid odontogenic tumor: report of an usually large lesion in the mandible. J Oral Maxillofac Surg 53: 714-717.

17. Takahashi K, Yoshino T, Hashimoto S (2001) Unsually large cystic adenomatoid odontogenic tumuor of the maxilla: case reposrt. Int J Oral Maxillofac Surg 30: 173-175.

18. Gadewar DR, Srikant N (2010) Adenomatoid odontogenic tumour: Tumour or a cyst, a histopathological support for the controversy. Int J Pediatr Otorhinolaryngol 74: 333-337.

19. Rick GM (2004) Adenomatoid odontogenic tumor. Oral Maxillofacial Surg Clin N Am 16: 333-354. 